\title{
Clinical Significance of Pleural Attachment and Indentation of Subsolid Nodule Lung Cancer
}

\author{
Hyung-Jun Kim, MD ${ }^{1}$ \\ Jun Yeun Cho, MD2,3 \\ Yeon Joo Lee, MD2,3 \\ Jong Sun Park, MD, PhD2,3 \\ Young-Jae Cho, MD, PhD 2,3 \\ Ho II Yoon, MD, PhD ${ }^{2,3}$ \\ Jin-Haeng Chung, MD, PhD ${ }^{4}$ \\ Sukki Cho, MD, PhD ${ }^{5}$ \\ Kwhanmien Kim, MD, PhD ${ }^{5}$ \\ Kyung Won Lee, MD, $\mathrm{PhD}^{6}$ \\ Jae Ho Lee, MD, $P h D^{2,3}$ \\ Choon-Taek Lee, MD, PhD 2,3
}

\begin{abstract}
${ }^{1}$ Department of Internal Medicine, Armed Forces Daegu Hospital, Gyeongsan, ${ }^{2}$ Division of Pulmonology and Critical Care Medicine, Department of Internal Medicine and Respiratory Center, Seoul National University Bundang Hospital, Seongnam, ${ }^{3}$ Division of Pulmonology and Critical Care Medicine, Department of Internal Medicine, Seoul National University College of Medicine, Seoul, Departments of ${ }^{4}$ Pathology, ${ }^{5}$ Thoracic and Cardiovascular Surgery, and ${ }^{6}$ Radiology, Seoul National University Bundang Hospital, Seongnam, Korea
\end{abstract}

\begin{abstract}
Purpose
Lung cancers presenting as subsolid nodule commonly have peripheral location, making the cancer-pleura relationship noteworthy. We aimed to evaluate the effect of pleural attachment and/or indentation on visceral pleural invasion (VPI) and recurrence-free survival.
\end{abstract}

\section{Materials and Methods}

Patients who underwent curative resection of lung cancer as subsolid nodules from April 2007 to January 2016 were retrospectively evaluated. They were divided into four groups according to their relationship with the pleura. Clinical, radiographical, and pathological findings were analyzed.

\section{Results}

Among 404 patients with malignant subsolid nodule, 120 (29.7\%) had neither pleural attachment nor indentation, 26 (6.4\%) had attachment only, 117 (29.0\%) had indentation only, and 141 (34.9\%) had both. VPI was observed in nodules of 36 patients (8.9\%), but absent in nonsolid nodules and in those without pleural attachment and/or indentation. Compared to subsolid nodules with concurrent pleural attachment and indentation, those with attachment only (odds ratio, 0.12; 95\% confidence interval [Cl], 0.02 to 0.98 ) and indentation only (odds ratio, $0.10 ; 95 \% \mathrm{Cl}, 0.03$ to 0.31 ) revealed lower odds of VPI. On subgroup analysis, the size of the solid portion was associated with VPI among those with pleural attachment and indentation ( $p=0.021$ ). Such high-risk features for VPI were associated with earlier lung cancer recurrence (adjusted hazard ratio, 3.31; 95\% Cl, 1.58 to 6.91).

\section{Conclusion}

Concurrent pleural attachment and indentation are risk factors for VPI, and the odds increase with larger solid portion in subsolid nodules. Considering the risk of recurrence, early surgical resection could be encouraged in these patients.
Correspondence: Choon-Taek Lee, MD, PhD Division of Pulmonology and Critical Care Medicine, Department of Internal Medicine and Respiratory Center, Seoul National University Bundang Hospital, 82 Gumi-ro 173 beon-gil, Bundang-gu, Seongnam 13620, Korea Tel: 82-31-787-7002

Fax: 82-31-787-4052

E-mail: ctlee@snu.ac.kr

Received January 25, 2019

Accepted March 22, 2019

Published Online March 25, 2019

\section{Key words}

Lung neoplasms, Non-small-cell lung carcinoma, Pleura,

Neoplasm invasiveness, Prognosis 


\section{Introduction}

Lung cancer is one of the most common cancers worldwide, with incidence of up to 20.0-44.0 per 100,000 persons in Northern America and Western Europe [1]. Mortality rates are high, and the 5-year survival rate is reportedly 10\%-20\% [2]. To decrease such lethal consequences, lung cancer screening using low-dose chest computed tomography (CT) in selected patients has proven to reduce overall mortality [3]. Furthermore, such screening has increased the detection of malignant subsolid nodules (SSNs) [4]. Such SSNs require regular follow-up because malignancy rates can be even higher for these nodules than for solid nodules [4]. Although the risk of recurrence after resection is low for subsolid lung cancer [5], the median survival after recurrence is expected to be $<3$ years [6]. Thus, identifying risk factors of recurrence is necessary for personalized treatment of patients.

Some factors, such as visceral pleural invasion (VPI), pathological tumor, node, and metastasis staging, lymphovascular invasion, and spread through air space, are associated with postoperative recurrence [7-9]. However, most of these factors can only be detected after a surgery is performed, making further resection difficult during surgery. Thus, it would be better if risk evaluation could be based on radiological findings performed before surgery.

SSNs, which are mostly composed of adenocarcinomas, are frequently found in the peripheral lungs. Therefore, the relationship between SSNs and the pleura is noteworthy. Linear pleural tags with soft tissue component at the pleural end on mediastinal window can predict VPI for non-small cell lung cancer (NSCLC) [10]. In contrast, studies of subsolid lung cancers have questioned the effect of indentation on VPI, although pleural contact is a significant factor of VPI $[11,12]$. The effect of pleura-SSN relationship on VPI, along with the effect on clinical prognoses, is not completely understood.

Therefore, this study aimed to evaluate the effect of pleural attachment and / or indentation on postoperative outcomes, including VPI and recurrence-free survival, in subsolid lung cancers.

\section{Materials and Methods}

\section{Study population}

Patients who underwent lung resection for SSNs from April 2007 to January 2016 in a tertiary referral center were screened. Among them, solitary lung cancer patients and those with surgery of curative intention were selected. Patients were divided into the following four groups based on CT findings before surgery: with or without pleural attachment and/or indentation.

\section{Patient assessment, treatment, and follow-up}

Baseline demographics, such as sex, age, smoking status, comorbidities, and pulmonary function test results, were acquired from medical records. The location of lung cancer was identified based on preoperative CT findings. Due to the retrospective nature of the study, the extent of surgery was determined by the attending surgeon. Postoperative adjuvant chemotherapy and / or radiotherapy were performed for high-risk patients with stage IB and IIA lung cancer or for those with pathological stage higher than IIB. After treatment, to detect recurrence, patients were followed up on 3-, 6 -, and 12-month intervals using chest CT.

\section{Radiographic evaluation}

Chest CT was performed using Brilliance 64 and iCT256 (Philips Medical System, Cleveland, OH). CT parameters were $120 \mathrm{kVp}$ and 100-200 mA, and images were reconstructed with a 1-3-mm thickness. All CT scans were obtained with the patient in the supine position at full inspiration, and the latest CT study obtained before surgical resection was selected for analysis. The mediastinal window had a level of 45 Hounsfield units (HUs) and a width of $250 \mathrm{HU}$, while the lung window had a level of $-500 \mathrm{HU}$ and a width of 2,000 HU.

SSNs were classified as nonsolid nodules (NSNs; formerly pure ground glass opacity nodule) if the opacity disappeared on the mediastinal window, and as part-solid nodules (PSNs; formerly mixed ground glass opacity nodule) if it persisted. According to the recent National Comprehensive Cancer Network (NCCN) guidelines, PSNs with solid component $\geq 8 \mathrm{~mm}$ are considered to have a higher probability of malignancy and those with solid component $\leq 5 \mathrm{~mm}$ are considered to have lower probability. Regarding such recommendation, PSNs were divided into three groups: PSN with solid portion $\leq 5 \mathrm{~mm}$, solid portion $>5 \mathrm{~mm}$ and $<8 \mathrm{~mm}$, and solid portion $\geq 8 \mathrm{~mm}$. The size of the solid portion was calculated as described in the NCCN guideline, i.e., by averaging the longest longitudinal length and largest diameter perpendicular to it.

Other radiographic findings, such as the longest diameter, distance from the pleura, pleural attachment, and indentation, were assessed in the lung window setting. Distance from the pleura was assessed by drawing a perpendicular line between the pleura and SSN, and the shortest distance was measured. SSN was defined to have a pleural attach- 


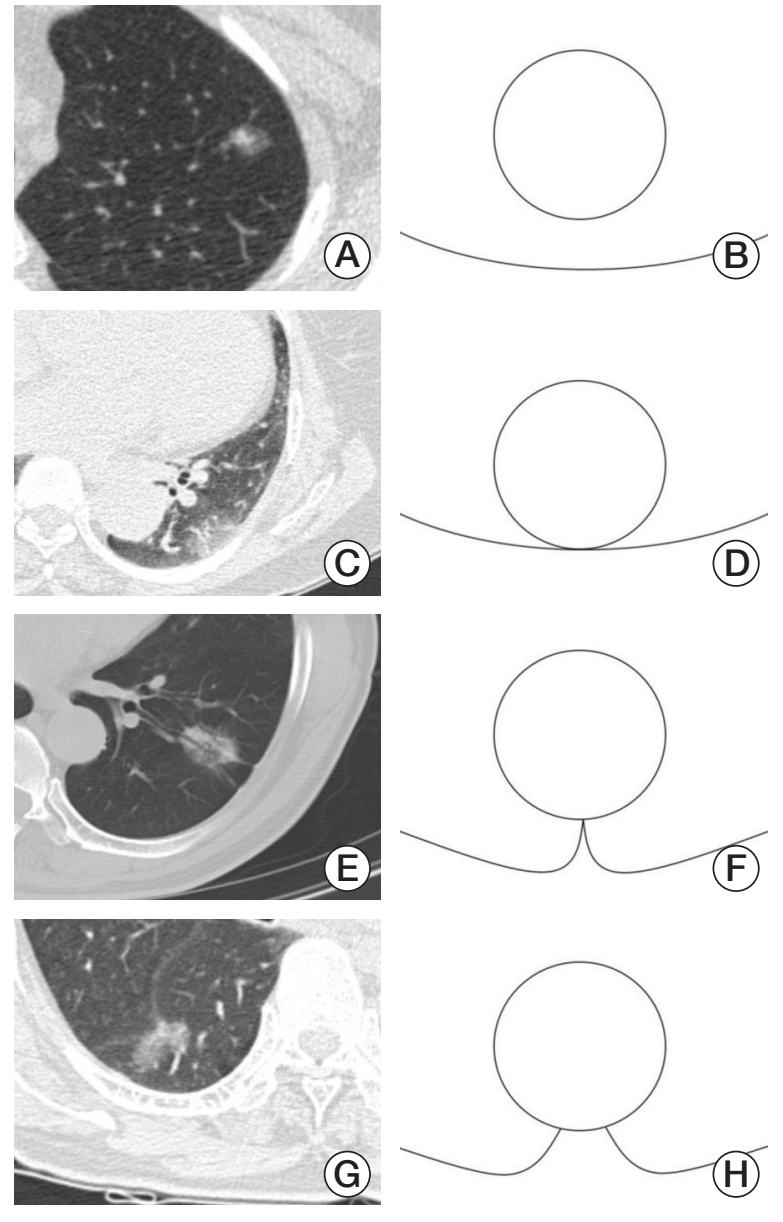

Fig. 1. Radiographic examples of subsolid nodules. (A, B) Without pleural attachment nor indentation. (C, D) With pleural attachment, but without indentation. (E, F) With indentation, but without pleural attachment. $(\mathrm{G}, \mathrm{H})$ With both pleural attachment and indentation.

ment when the distance from the pleura was $0 \mathrm{~mm}$. Pleural indentation was defined as linear areas of high attenuation originating from the SSN extending peripherally to contact the pleura [13,14]. When SSN was attached to the pleura, any distortions of the pleural architecture by SSN was also defined as pleural indentation and then defined as concurrent pleural attachment with indentation [12]. SSNs were divided into four groups according to the relationship with the pleura: neither pleural attachment nor indentation, pleural attachment without indentation, pleural indentation without attachment, and concurrent pleural attachment with indentation. The interpretation of the CT findings was done by an investigator who was not aware of any clinical information. The examples of the four groups are shown in Fig. 1.

\section{Pathological evaluation}

Pathological staging was retrospectively determined according to the eighth edition of the American Joint Committee on Cancer (AJCC) staging system [15]. T category was described in eight categories: $\mathrm{T} 1 \mathrm{a}(\mathrm{mi})$ to $\mathrm{T} 4$; and $\mathrm{N}$ category in three categories: N0 to N2. When a lymph node was not evaluated either preoperatively or intraoperatively, it was considered Nx. The overall stage grouping was specified in eight categories (from IA1 to IIIB), in which stage IIIC and IV cancers were not included. Specimens obtained in the early phase of the study were reviewed to allocate them into proper stage according to the AJCC eighth edition. Pathological VPI was considered to be present when invasion beyond the elastic layer was observed [16]. Other pathological findings, such as vascular invasion and lymphatic invasion, were also recorded. An investigator blinded from any clinical information participated in the analysis of the pathological findings.

\section{Statistical analysis}

Baseline demographics and CT, pathological, and clinical findings were organized as numbers (\%) for categorical variables using the chi-square test or Fisher exact test. For continuous variables, Student's $\mathrm{t}$ test or Mann-Whitney U test was performed to describe the values in mean \pm standard variation or median (interquartile range [IQR]) according to their patterns of distribution. The pathological stage was grouped as T1, T2, and T3-4 for T category and as I, II, and III for overall stage group to compare the characteristics between patients with or without pleural attachment and / or indentation. Multivariate logistic regression analysis was performed to measure the odds ratios (ORs) and 95\% percentile confidence interval $(\mathrm{CI})$ of important radiographic predictors of VPI.

Furthermore, survival analysis was performed with KaplanMeier curve with log-rank test and Cox proportional hazards models to evaluate predictors of lung cancer recurrence. Variables with $p$-values of $<0.1$ were included in the multivariate Cox regression analysis. Hazard ratios (HRs) with 95\% CI were calculated.

All statistical analyses were performed using Stata ver. 13.0 (Stata Corp., College Station, TX). p-values $<0.05$ were considered statistically significant.

\section{Ethical statement}

This study was conducted according to the amended Declaration of Helsinki and was approved by the institutional review board of Seoul National University Bundang Hospital (protocol number: B-1604-343-104). Informed consent was 
Table 1. Baseline characteristics of 404 patients who underwent curative surgery for subsolid nodule

\begin{tabular}{|c|c|c|c|c|c|}
\hline Variable & $\begin{array}{c}A-I- \\
(n=120)\end{array}$ & $\begin{array}{l}A+I- \\
(n=26)\end{array}$ & $\begin{array}{c}A-I+ \\
(n=117)\end{array}$ & $\begin{array}{c}A+I+ \\
(n=141)\end{array}$ & p-value \\
\hline Male & $56(46.7)$ & $12(46.2)$ & $63(53.9)$ & $56(39.7)$ & 0.162 \\
\hline Age (yr) & $60.3 \pm 10.4$ & $63.8 \pm 9.3$ & $64.0 \pm 10.5$ & $63.8 \pm 9.8$ & 0.015 \\
\hline Ever smoker & $40(33.3)$ & $11(42.3)$ & $50(42.7)$ & $48(34.0)$ & 0.364 \\
\hline \multicolumn{6}{|l|}{ Comorbidity } \\
\hline Other malignancy in $5 \mathrm{yr}$ & $15(12.5)$ & $2(7.7)$ & $14(12.0)$ & $18(12.8)$ & 0.907 \\
\hline History of tuberculosis & $2(1.7)$ & $4(15.4)$ & $9(7.7)$ & $4(2.8)$ & 0.008 \\
\hline Asthma & $3(2.5)$ & 0 & $3(2.6)$ & $3(2.1)$ & $>0.999$ \\
\hline Bronchiectasis & 0 & $1(3.9)$ & $3(2.6)$ & $4(2.8)$ & 0.170 \\
\hline COPD & 0 & 0 & $1(0.9)$ & $2(1.4)$ & 0.710 \\
\hline \multicolumn{6}{|l|}{ Preoperative PFT results } \\
\hline FVC (L) & $3.48 \pm 0.77$ & $3.07 \pm 0.73$ & $3.41 \pm 0.89$ & $3.18 \pm 0.77$ & 0.005 \\
\hline $\mathrm{FEV}_{1}(\mathrm{~L})$ & $2.65 \pm 0.57$ & $2.39 \pm 0.55$ & $2.55 \pm 0.74$ & $2.42 \pm 0.58$ & 0.018 \\
\hline $\mathrm{FEV}_{1} / \mathrm{FVC}(\%)$ & $76.7 \pm 8.2$ & $78.2 \pm 5.9$ & $74.9 \pm 10.1$ & $76.6 \pm 9.2$ & 0.240 \\
\hline \multicolumn{6}{|l|}{ Location of lung cancer } \\
\hline Right upper lobe & $53(44.2)$ & $6(23.1)$ & $44(37.6)$ & $50(35.5)$ & 0.045 \\
\hline Right middle lobe & $2(1.7)$ & $1(3.9)$ & $3(2.6)$ & $15(10.6)$ & \\
\hline Right lower lobe & $22(18.3)$ & $6(23.1)$ & $24(20.5)$ & $21(14.9)$ & \\
\hline Left upper lobe & $30(25.0)$ & $6(23.1)$ & $26(22.2)$ & $32(22.7)$ & \\
\hline Left lower lobe & $13(10.8)$ & $7(26.9)$ & $20(17.1)$ & $23(16.3)$ & \\
\hline \multicolumn{6}{|l|}{ Type of subsolid nodule } \\
\hline Nonsolid nodule & $61(50.8)$ & $4(15.4)$ & $18(15.4)$ & $26(18.4)$ & $<0.001$ \\
\hline \multicolumn{6}{|l|}{ Part-solid nodule } \\
\hline Solid portion $\leq 5 \mathrm{~mm}$ & $31(25.8)$ & $3(11.5)$ & $13(11.1)$ & $19(13.5)$ & \\
\hline Solid portion $5-8 \mathrm{~mm}^{\mathrm{a}}$ & $14(11.7)$ & $3(11.5)$ & $13(11.1)$ & $17(12.1)$ & \\
\hline Solid portion $\geq 5 \mathrm{~mm}$ & $14(11.7)$ & $16(61.5)$ & $73(62.4)$ & $79(56.0)$ & \\
\hline Longest diameter, $\mathrm{mm}$ & $16.7 \pm 5.7$ & $22.7 \pm 9.6$ & $23.9 \pm 9.7$ & $24.8 \pm 14.0$ & $<0.001$ \\
\hline \multicolumn{6}{|l|}{ Extent of surgery } \\
\hline Wedge resection & $33(27.5)$ & $7(26.9)$ & $20(17.1)$ & $32(22.7)$ & 0.319 \\
\hline Segmentectomy & $17(14.2)$ & $4(15.4)$ & $20(17.1)$ & $14(9.9)$ & \\
\hline Lobectomy & $69(57.5)$ & $15(57.7)$ & $77(65.8)$ & $95(67.4)$ & \\
\hline Pneumonectomy & $1(0.8)$ & 0 & 0 & 0 & \\
\hline \multicolumn{6}{|l|}{ Adjuvant therapy } \\
\hline None & $118(98.3)$ & $26(100)$ & $109(93.2)$ & $124(87.9)$ & 0.012 \\
\hline Chemotherapy & $2(1.7)$ & 0 & $5(4.3)$ & $15(10.6)$ & \\
\hline Concurrent chemo-radiation & 0 & 0 & $3(2.6)$ & $2(1.4)$ & \\
\hline
\end{tabular}

Values are presented as number $(\%)$ or mean \pm standard variation. A, attachment; I, indentation; COPD, chronic obstructive pulmonary disease; PFT, pulmonary function test; FVC, forced vital capacity; $\mathrm{FEV}_{1}$, forced expiratory volume in 1 second. ${ }^{a)}$ Excludes 5 and $8 \mathrm{~mm}$.

waived because of the retrospective design of the study; however, records and information of each patient were anonymized and de-identified before analysis.

\section{Results}

\section{Patient selection and baseline characteristics}

During the 9-year study period, 541 patients underwent lung resection surgery for SSNs, of whom, patients with multiple primary lung cancer $(n=73)$, benign lesion $(n=23)$, adenocarcinoma in situ $(\mathrm{n}=20)$, recurred case $(\mathrm{n}=8)$, malignancy 
Table 2. Radiographic findings associated with visceral pleural invasion

\begin{tabular}{|c|c|c|c|}
\hline Variable & VPI present $(\mathrm{n}=36)$ & VPI absent $(n=368)$ & p-value \\
\hline \multicolumn{4}{|l|}{ Relation with pleura } \\
\hline $\mathrm{A}(-) \mathrm{I}(-)$ & $0 / 120(0)$ & $120 / 120(100)$ & $<0.001$ \\
\hline $\mathrm{A}(+) \mathrm{I}(-)$ & $1 / 26(3.9)$ & $25 / 26(96.2)$ & \\
\hline $\mathrm{A}(-) \mathrm{I}(+)$ & 4/117 (3.4) & $113 / 117(96.6)$ & \\
\hline $\mathrm{A}(+) \mathrm{I}(+)$ & $31 / 141(22.0)$ & $110 / 141(78.0)$ & \\
\hline \multicolumn{4}{|l|}{ Type of subsolid nodule } \\
\hline Nonsolid nodule & 0/109(0) & 109/109 (100) & $<0.001$ \\
\hline \multicolumn{4}{|l|}{ Part-solid nodule } \\
\hline Solid portion $\leq 5 \mathrm{~mm}$ & $1 / 66(1.5)$ & $65 / 66$ (98.5) & \\
\hline Solid portion $5-8 \mathrm{~mm}^{\mathrm{a})}$ & $3 / 47(6.4)$ & $44 / 47(93.6)$ & \\
\hline Solid portion $\geq 8 \mathrm{~mm}$ & 32/182 (17.5) & $150 / 182(82.4)$ & \\
\hline Longest diameter (mm) & $27(23-40)$ & $19(14-25)$ & $<0.001$ \\
\hline \multicolumn{4}{|l|}{ Location of lung cancer } \\
\hline Right upper lobe & $10 / 153(6.5)$ & $143 / 153(93.5)$ & 0.694 \\
\hline Right middle lobe & 3/21 (14.2) & $18 / 21(85.7)$ & \\
\hline Right lower lobe & $7 / 73(9.6)$ & $66 / 73(90.4)$ & \\
\hline Left upper lobe & 10/94 (10.6) & $84 / 94(89.4)$ & \\
\hline Left lower lobe & $6 / 63(9.5)$ & $57 / 63(90.5)$ & \\
\hline
\end{tabular}

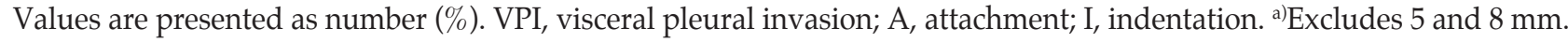

other than lung cancer $(n=5)$, distant metastasis $(n=4)$, follow-up loss $(\mathrm{n}=2)$, N3 disease $(\mathrm{n}=1)$, and other coexisting malignancies in the resected specimen $(\mathrm{n}=1)$ were excluded. Finally, 404 patients were included. Neither pleural attachment nor indentation was observed in 120 patients $(29.7 \%)$, pleural attachment without indentation in $26(6.4 \%)$, pleural indentation without attachment in 117 (29.0\%), and concurrent pleural attachment with indentation in 141 (34.9\%). After surgery, pathological VPI was proven in 36 patients $(8.9 \%)$. Thirty-four patients $(8.4 \%)$ experienced lung cancer recurrence at the point of review (S1 Fig.).

Patients had a fairly even distribution of sex, with a mean age of 62.8 years. Patients without pleural attachment or attachment were younger than others $(\mathrm{p}=0.015)$. More than half of the patients $(63.3 \%)$ were never smokers, with common comorbidities of hypertension (29.2\%), diabetes mellitus $(13.1 \%)$, and other malignancies within 5 years $(12.1 \%)$. Chronic lung diseases, such as asthma $(n=9)$, bronchiectasis $(\mathrm{n}=8)$, and chronic obstructive pulmonary disease $(\mathrm{n}=3)$, were observed in 19 patients (4.7\%). Subsolid lung cancer was the commonest in the right upper lobe (38.9\%), and concurrent pleural attachment with indentation was predominantly observed in the right middle lobe $(10.6 \%)$. Pleural attachment and/or indentation were observed more frequently among those with PSNs and those with longer diameter. The extent of lung resection was not significantly different among the four groups $(\mathrm{p}=0.319)$, with lobectomy being the most common surgery performed (257 patients,
$63.4 \%)$. Compared to sublobar resection such as wedge resection and segmentectomy, lobectomy was performed in patients with larger solid portion $(p<0.001)$, longer longitudinal diameter (median, $24 \mathrm{~mm}$ vs. $15 \mathrm{~mm} ; \mathrm{p}<0.001$ ), and when the SSN was located in right upper lobe or right middle lobe $(p<0.001)$. Furthermore, patients who underwent lobectomy revealed higher rates of VPI compared to those of sublobar resection ( $11.3 \%$ vs. $4.8 \%, \mathrm{p}=0.026)$, along with higher overall pathological stage $(\mathrm{p}<0.001)$. Adjuvant therapy was performed in 27 of 404 patients $(6.7 \%)$, which were more frequently performed among those with pleural attachment and indentation (7 among 141 patients, 12.0\%) (Table 1).

\section{Radiographic characteristics and pathological correlation}

Pathology reports of postoperative lung specimen were reviewed. All patients were diagnosed with adenocarcinoma, including invasive adenocarcinoma $(\mathrm{n}=332,82.2 \%)$ and minimally invasive adenocarcinoma $(\mathrm{n}=72,17.8 \%)$. VPI was observed in 36 patients $(8.9 \%)$. SSNs with pleural attachment and indentation had higher association with VPI (31 of 141 patients, $22.0 \%$ ) than those with attachment alone (1 of 26 patients, 3.9\%), indentation alone (4 of 117 patients, 3.4\%), and neither ( 0 of 120 patients, $0.0 \%)(\mathrm{p}<0.001)$. None of the NSNs had VPI, and those with larger solid portion $(p<0.001)$ and longer diameter (median, $27 \mathrm{~mm}$ vs. $19 \mathrm{~mm}$; $\mathrm{p}<0.001$ ) had a higher probability of VPI (Table 2). Multivariate logistic regression analysis was performed with meaningful factors associ- 
Table 3. Radiographic factors associated with visceral pleural invasion in subsolid lung adenocarcinoma

\begin{tabular}{|c|c|c|c|}
\hline Variable & OR & $95 \% \mathrm{CI}$ & p-value \\
\hline \multicolumn{4}{|l|}{ Relation with pleura } \\
\hline $\mathrm{A}(-) \mathrm{I}(-)$ & $\mathrm{N} / \mathrm{A}$ & $\mathrm{N} / \mathrm{A}$ & $\mathrm{N} / \mathrm{A}$ \\
\hline $\mathrm{A}(+) \mathrm{I}(-)$ & 0.12 & $0.02-0.98$ & 0.048 \\
\hline $\mathrm{A}(-) \mathrm{I}(+)$ & 0.10 & $0.03-0.31$ & $<0.001$ \\
\hline $\mathrm{A}(+) \mathrm{I}(+)$ & Reference & & \\
\hline \multicolumn{4}{|l|}{ Size of the solid portion } \\
\hline Absent solid portion & $\mathrm{N} / \mathrm{A}$ & $\mathrm{N} / \mathrm{A}$ & $\mathrm{N} / \mathrm{A}$ \\
\hline Solid portion $\leq 5 \mathrm{~mm}$ & 0.13 & $0.02-1.02$ & 0.052 \\
\hline Solid portion $5-8 \mathrm{~mm}^{\mathrm{a})}$ & 0.44 & $0.12-1.65$ & 0.221 \\
\hline Solid portion $\geq 8 \mathrm{~mm}$ & Reference & & \\
\hline Longest diameter, by $\mathrm{cm}$ & 1.24 & $0.93-1.64$ & 0.144 \\
\hline
\end{tabular}

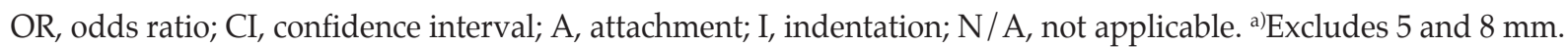

Table 4. Size of the solid portion and visceral pleural invasion according to the pleura-nodule relationship

\begin{tabular}{|c|c|c|c|c|c|}
\hline \multirow{2}{*}{ Variable } & \multicolumn{4}{|c|}{ Solid portion } & \multirow{2}{*}{ p-value } \\
\hline & None & $\leq 5 \mathrm{~mm}$ & 5-8 $\mathrm{mm}^{\mathrm{a})}$ & $\geq 8 \mathrm{~mm}$ & \\
\hline VPI in $\mathrm{A}(-) \mathrm{I}(-)$ group & $0 / 61(0)$ & $0 / 31(0)$ & $0 / 14(0)$ & $0 / 14(0)$ & $\mathrm{N} / \mathrm{A}$ \\
\hline VPI in $\mathrm{A}(+) \mathrm{I}(-)$ group & $0 / 4(0)$ & $0 / 3(0)$ & $0 / 3(0)$ & $1 / 16(6.3)$ & $>0.999$ \\
\hline VPI in $\mathrm{A}(-) \mathrm{I}(+)$ group & $0 / 18(0)$ & $0 / 13(0)$ & $0 / 13(0)$ & $4 / 73(5.5)$ & 0.849 \\
\hline VPI in $\mathrm{A}(+) \mathrm{I}(+)$ group & $0 / 26(0)$ & $1 / 19(5.3)$ & $3 / 17(17.6)$ & $27 / 79(34.1)$ & $<0.001$ \\
\hline
\end{tabular}

Values are presented as number (\%). VPI, visceral pleural invasion; A, attachment; I, indentation; N/A, not available. ${ }^{\text {a)}}$ Excludes 5 and $8 \mathrm{~mm}$.

ated with VPI. Compared with SSNs with concurrent pleural attachment and indentation, those with attachment only (OR, $0.12 ; 95 \% \mathrm{CI}, 0.02$ to 0.98 ) and indentation only (OR, 0.10; $95 \%$ CI, 0.03 to 0.31 ) revealed lower odds of VPI (Table 3 ).

Pathological staging according to the recent eighth AJCC recommendation was retrospectively performed. The pathological $\mathrm{T}$ category was higher among those with pleural attachment and/or indentation. Approximately 31.9\% of the patients with concurrent pleural attachment and indentation had at least T2 category, while only two patients (3.4\%) with neither pleural attachment nor indentation had T2 category $(\mathrm{p}<0.001)$. Vascular and lymphatic invasions were also more frequently observed in patients with pleural indentation than in those without it. Although pathological N category $(p=0.235)$ did not reveal statistical difference according to pleural attachment and/or indentation, the overall pathological stage was higher with pleural attachment and indentation $(\mathrm{p}=0.038)$ (S2 Table).

Subgroup analysis of patients with the four different features of SSN-pleura relationship was performed, and the probability of VPI was calculated according to the size of the solid portion in each group. The size of the solid portion larger than $5 \mathrm{~mm}$ was associated with VPI among PSNs with concurrent pleural attachment and indentation ( $<<0.001)$, while it was not associated with VPI among other groups (Table 4). Patients with concurrent pleural attachment and indentation, along with solid portion $>5 \mathrm{~mm}$, were categorized as "high risk of VPI" in the following survival analysis.

\section{Differences in clinical outcomes}

The median follow-up duration for the 404 patients was 48.3 months (IQR, 31.2 to 61.2). Clinical outcomes, such as lung cancer recurrence, and all-cause mortality were compared. Thirty-four patients $(8.4 \%)$ experienced recurrence within a median of 26.2 months (IQR, 11.7 to 38.5), and 19 patients $(4.7 \%)$ died in a median of 38.2 months (IQR, 24.4 to 50.1). Concurrent pleural attachment with indentation was associated with higher recurrence $(15.6 \%)$ and all-cause mortality rate $(7.1 \%)$ than those without any one of them $(3.3 \%$ and $0.8 \%$, respectively).

Recurrence-free survival (RFS) was compared with Kaplan- 


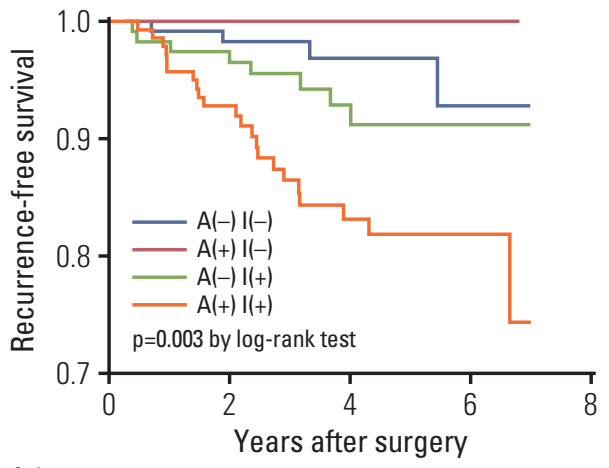

No. at risk

$\begin{array}{rrrrrr}A(-) I(-) & 120 & 102 & 53 & 12 & 0 \\ A(+) I(-) & 26 & 15 & 6 & 3 & 0 \\ A(-) I(+) & 117 & 105 & 57 & 11 & 0 \\ A(+) I(+) & 141 & 115 & 65 & 20 & 0\end{array}$

Fig. 2. Effect of pleural attachment and indentation on recurrence-free survival of subsolid lung adenocarcinoma. A, attachment; I, indentation.

Meier curve and log-rank test, which revealed that concurrent pleural attachment with indentation was associated with shorter time to recurrence in the subsolid lung cancers than in other groups (Fig. 2). With multivariate Cox proportional hazards model, smoking history (adjusted HR, 2.20; 95\% CI, 1.11 to 4.35), "high risk of VPI" features (adjusted HR, 3.31; 95\% CI, 1.58 to 6.91), and longer diameter (adjusted HR, 1.42; 95\% CI, 1.21 to 1.67 per $\mathrm{cm}$ ) were significantly associated with earlier time to recurrence (S3 Table).

\section{Discussion}

In this retrospective cohort study, pleural attachment and/or indentation was observed in more than two-thirds (284 patients) of the 404 patients who underwent curative surgery for subsolid lung cancer. Subsolid lung cancers with pleural attachment and/or indentation were observed in patients with older age and those with larger solid portion and longer diameter. Pleural attachment and/or indentation was not associated with the extent of surgery but was associated with more frequent adjuvant therapies. VPI was not observed in NSNs, while concurrent pleural attachment with indentation was assessed as an independent factor associated with VPI. The size of the solid portion $>5 \mathrm{~mm}$ was associated with higher risk of VPI among patients with concurrent pleural attachment and indentation. Time to lung cancer recur- rence was shorter among patients with concurrent pleural attachment and indentation and solid portion $>5 \mathrm{~mm}$.

In this study, the rates of pleural attachment $(41.3 \%)$, indentation $(63.9 \%)$, and VPI (8.9\%) were comparable with those reported previously. Pleural attachment was reportedly present in 35.3\%-62.2\% and indentation in 19.8\%-66.4\% among subsolid lung adenocarcinomas [11,12]. The incidence of VPI among nonsolid lung adenocarcinomas is reportedly $0.0 \%-17.4 \%$ and that among part-solid lung adenocarcinoma is $10.0 \%-32.2 \%[7,11,12]$. We found no VPI among NSNs but found $12.2 \%$ of VPI among PSNs.

This is the first study to demonstrate that concurrent pleural attachment with indentation was independently associated with VPI among subsolid lung cancers. Compared to simple attachment of SSN to the pleura, further indentation to the pleura is caused by a combination of two stepwise consequences: contractile change of the cancer and a compensatory enlargement of surrounding tissues to fill the territory between the areas of retracted visceral pleura [17]. Therefore, there exists a higher possibility that the cancer invaded the point of tagged visceral pleura. Pleural indentation has been universally considered a positive predictive factor for VPI and lymphovascular invasiveness in NSCLC, including solid cancers $[10,18]$. However, considering that chronic lung diseases, such as tuberculosis-destroyed lung, bronchiectasis, and chronic obstructive pulmonary disease, may cause architectural distortion of the lung anatomy, indentation alone may not be relied upon as an independent risk factor for VPI, as shown previously [11]. Furthermore, most previous studies were focused on solid lung cancers. Subsolid lung cancers are considered to be less invasive compared to solid tumors and should be examined separately from solid lung cancers [5]. The importance of concurrent pleural attachment and indentation was previously suggested in a retrospective study of 194 NSCLC patients [19]. Pleural indentation with other additional relationships to the pleura leads to poorer outcomes compared with those with sole indentation.

In a subgroup of subsolid lung cancers with concurrent pleural attachment and indentation, larger solid portion ( $>5$ $\mathrm{mm}$ ) was significantly associated with higher odds of VPI. A larger solid portion was reportedly an independent factor of VPI among PSNs, and NSNs do not show VPI, regardless of their relationship with the pleura [11,20,21]. The relationship with the pleura, presented as concurrent pleural attachment with indentation, should be emphasized as an independent factor for VPI, and if the solid portion is larger, a higher risk of VPI should be suspected.

In our study population, $8.4 \%$ of patients experienced recurrence of subsolid lung adenocarcinoma during a median 48.3 months of follow-up. The calculated 5-year RFS was $94.4 \%$ for patients with stage IA and $69.3 \%$ for those with stage higher stages. The RFS rates of stage IA are comparable 
with those reported in the previous study of subsolid lung adenocarcinomas [7].

VPI is a well-known significant risk factor for recurrence and poor outcomes of NSCLC [22-24]. In subsolid lung cancer, however, the effect of VPI on prognosis is still debated. From a retrospective study of 147 subsolid lung cancers without nodal metastasis, VPI was associated with RFS [7], but another retrospective study of 466 patients demonstrated similar RFS between the two groups [25]. In our study, pathological VPI was an independent factor for earlier recurrence of subsolid lung cancer (adjusted HR, 3.88; 95\% CI, 1.84 to $8.15)$, and "high risk of VPI" feature, defined as concurrent pleural attachment and indentation with solid portion $>5$ $\mathrm{mm}$, was independently associated with earlier recurrence. Pleural indentation is associated with poor outcome among NSCLC [19]. Indentation increases the risk of invasion to the visceral pleura, in which lymphatic drainage is abundant, leading to a higher risk of tumor dissemination [26]. Pleural attachment is another known factor for locoregional recurrence after radiotherapy in NSCLC and poor survival in lung adenocarcinoma $[27,28]$. Therefore, the "high risk of VPI" feature is associated with higher risk of VPI, and it also increases the risk of recurrence, leading to poor prognosis after surgical resection. The recent NCCN guidelines for lung cancer screening and the statement from the Fleischner Society have also emphasized the importance of the size of the solid area $>5 \mathrm{~mm}$ as a higher risk of malignancy among subsolid lung cancers [29].

Despite the important findings above, this study had some limitations. First, this was a retrospective study performed at a single center. More studies from different centers are required for generalizability of the results. Second, we did not differentiate pleural invasion beyond the elastic layer (PL1) and invasion to the visceral pleural surface (PL2).
Because the discrimination of PL1 and PL2 was performed at our center after September 2012, patients who underwent surgery before that time did not have appropriate pathological reports regarding the two. It must be recognized that prognostic difference may exist between PL1 and PL2 disease [30]. Third, the study period was long (9 years). The changing lung cancer staging systems and standards of care may have affected the recurrence rates. Furthermore, the follow-up durations of patients differ according to the time of the operation.

In conclusion, concurrent pleural attachment with indentation of subsolid lung cancer is an independent risk factor for VPI, and the odds of VPI were higher among those with solid portion $>5 \mathrm{~mm}$. Furthermore, patients with such "high risk of VPI" features experienced earlier recurrence. Therefore, such high-risk findings should be recognized on preoperative CT scans, and earlier surgery could be recommended in these selected patients.

\section{Electronic Supplementary Material}

Supplementary materials are available at Cancer Research and Treatment website (https: // www.e-crt.org).

\section{Conflicts of Interest}

Conflict of interest relevant to this article was not reported.

\section{Acknowledgments}

We deeply appreciate the members of the Department of Internal Medicine, Seoul National University College of Medicine for sharing their ideas and comments.

This study was supported by SNUBH grant (21-2018-055).

\section{References}

1. Torre LA, Bray F, Siegel RL, Ferlay J, Lortet-Tieulent J, Jemal A. Global cancer statistics, 2012. CA Cancer J Clin. 2015;65:87108.

2. Allemani C, Weir HK, Carreira H, Harewood R, Spika D, Wang XS, et al. Global surveillance of cancer survival 19952009: analysis of individual data for $25,676,887$ patients from 279 population-based registries in 67 countries (CONCORD2). Lancet. 2015;385:977-1010.

3. National Lung Screening Trial Research Team, Aberle DR, Adams AM, Berg CD, Black WC, Clapp JD, et al. Reduced lung-cancer mortality with low-dose computed tomographic screening. N Engl J Med. 2011;365:395-409.
4. Henschke CI, Yankelevitz DF, Mirtcheva R, McGuinness G, McCauley D, Miettinen OS, et al. CT screening for lung cancer: frequency and significance of part-solid and nonsolid nodules. AJR Am J Roentgenol. 2002;178:1053-7.

5. Nakamura S, Fukui T, Kawaguchi K, Fukumoto K, Hirakawa A, Yokoi K. Does ground glass opacity-dominant feature have a prognostic significance even in clinical T2aN0M0 lung adenocarcinoma? Lung Cancer. 2015;89:38-42.

6. Ujiie H, Kadota K, Chaft JE, Buitrago D, Sima CS, Lee MC, et al. Solid predominant histologic subtype in resected stage I lung adenocarcinoma is an independent predictor of early, extrathoracic, multisite recurrence and of poor postrecurrence 
survival. J Clin Oncol. 2015;33:2877-84.

7. Seok Y, Lee E. Visceral pleural invasion is a significant prognostic factor in patients with partly solid lung adenocarcinoma sized $30 \mathrm{~mm}$ or smaller. Thorac Cardiovasc Surg. 2018;66: 150-5.

8. Kadota K, Nitadori J, Sima CS, Ujiie H, Rizk NP, Jones DR, et al. Tumor spread through air spaces is an important pattern of invasion and impacts the frequency and location of recurrences after limited resection for small stage I lung adenocarcinomas. J Thorac Oncol. 2015;10:806-14.

9. Lu S, Tan KS, Kadota K, Eguchi T, Bains S, Rekhtman N, et al. Spread through Air Spaces (STAS) is an independent predictor of recurrence and lung cancer-specific death in squamous cell carcinoma. J Thorac Oncol. 2017;12:223-34.

10. Hsu JS, Han IT, Tsai TH, Lin SF, Jaw TS, Liu GC, et al. Pleural tags on CT scans to predict visceral pleural invasion of nonsmall cell lung cancer that does not abut the pleura. Radiology. 2016;279:590-6.

11. Ahn SY, Park CM, Jeon YK, Kim H, Lee JH, Hwang EJ, et al. Predictive CT features of visceral pleural invasion by T1-sized peripheral pulmonary adenocarcinomas manifesting as subsolid nodules. AJR Am J Roentgenol. 2017;209:561-6.

12. Zhao LL, Xie HK, Zhang LP, Zha JY, Zhou FY, Jiang GN, et al. Visceral pleural invasion in lung adenocarcinoma $\leq 3 \mathrm{~cm}$ with ground-glass opacity: a clinical, pathological and radiological study. J Thorac Dis. 2016;8:1788-97.

13. Zwirewich CV, Vedal S, Miller RR, Muller NL. Solitary pulmonary nodule: high-resolution CT and radiologic-pathologic correlation. Radiology. 1991;179:469-76.

14. Ko JP, Suh J, Ibidapo O, Escalon JG, Li J, Pass H, et al. Lung adenocarcinoma: correlation of quantitative CT findings with pathologic findings. Radiology. 2016;280:931-9.

15. Goldstraw P, Chansky K, Crowley J, Rami-Porta R, Asamura $\mathrm{H}$, Eberhardt WE, et al. The IASLC Lung Cancer Staging Project: proposals for revision of the TNM stage groupings in the forthcoming (Eighth) edition of the TNM classification for lung cancer. J Thorac Oncol. 2016;11:39-51.

16. Travis WD, Giroux DJ, Chansky K, Crowley J, Asamura H, Brambilla E, et al. The IASLC Lung Cancer Staging Project: proposals for the inclusion of broncho-pulmonary carcinoid tumors in the forthcoming (seventh) edition of the TNM Classification for Lung Cancer. J Thorac Oncol. 2008;3:1213-23.

17. Seki N, Fujita Y, Shibakuki R, Seto T, Uematsu K, Eguchi K. Easier understanding of pleural indentation on computed tomography. Intern Med. 2007;46:2029-30.

18. Shimada Y, Yoshida J, Hishida T, Nishimura M, Ishii G, Nagai K. Predictive factors of pathologically proven noninvasive tumor characteristics in T1aN0M0 peripheral non-small cell lung cancer. Chest. 2012;141:1003-9.

19. Meniga IN, Tiljak MK, Ivankovic D, Aleric I, Zekan M, Hrabac $\mathrm{P}$, et al. Prognostic value of computed tomography morphologic characteristics in stage I non-small-cell lung cancer. Clin Lung Cancer. 2010;11:98-104.

20. Zhang H, Lu C, Lu Y, Yu B, Lv F, Zhu Z. The predictive and prognostic values of factors associated with visceral pleural involvement in resected lung adenocarcinomas. Onco Targets Ther. 2016;9:2337-48.

21. Bak SH, Lee HY, Kim JH, Um SW, Kwon OJ, Han J, et al. Quantitative CT scanning analysis of pure ground-glass opacity nodules predicts further CT scanning change. Chest. 2016;149:180-91.

22. Jiang L, Liang W, Shen J, Chen $X$, Shi $X, H e ~ J$, et al. The impact of visceral pleural invasion in node-negative non-small cell lung cancer: a systematic review and meta-analysis. Chest. 2015;148:903-11.

23. Huang H, Wang T, Hu B, Pan C. Visceral pleural invasion remains a size-independent prognostic factor in stage I nonsmall cell lung cancer. Ann Thorac Surg. 2015;99:1130-9.

24. Liu QX, Deng XF, Zhou D, Li JM, Min JX, Dai JG. Visceral pleural invasion impacts the prognosis of non-small cell lung cancer: a meta-analysis. Eur J Surg Oncol. 2016;42:1707-13.

25. Hattori A, Suzuki K, Matsunaga T, Takamochi K, Oh S. Visceral pleural invasion is not a significant prognostic factor in patients with a part-solid lung cancer. Ann Thorac Surg. 2014; 98:433-8.

26. Antony VB, Sahn SA, Mossman B, Gail DB, Kalica A. NHLBI workshop summaries. Pleural cell biology in health and disease. Am Rev Respir Dis. 1992;145:1236-9.

27. Yamamoto T, Kadoya N, Shirata Y, Koto M, Sato K, Matsushita $\mathrm{H}$, et al. Impact of tumor attachment to the pleura measured by a pretreatment CT image on outcome of stage I NSCLC treated with stereotactic body radiotherapy. Radiat Oncol. 2015;10:35.

28. Wang H, Schabath MB, Liu Y, Berglund AE, Bloom GC, Kim $\mathrm{J}$, et al. Semiquantitative computed tomography characteristics for lung adenocarcinoma and their association with lung cancer survival. Clin Lung Cancer. 2015;16:e141-63.

29. Naidich DP, Bankier AA, MacMahon H, Schaefer-Prokop CM, Pistolesi M, Goo JM, et al. Recommendations for the management of subsolid pulmonary nodules detected at CT: a statement from the Fleischner Society. Radiology. 2013;266:304-17.

30. Wang T, Zhou C, Zhou Q. Extent of visceral pleural invasion affects prognosis of resected non-small cell lung cancer: a meta-analysis. Sci Rep. 2017;7:1527. 